\title{
EFFECTIVENESS OF SUPERVISED TOOTH BRUSHING EXERCISE ON THE ORAL HYGIENE STATUS OF SCHOOL GOING CHILDREN IN PESHAWAR
}

\author{
Samrina Muhammad, Rubab Jawed*, Zeeshan Kibria**, Shazia Makdoom, Aiman Khan, Momina Saleem \\ Khyber College of Dentistry, Peshawar Pakistan, *Baqai Dental College, Karachi Pakistan, **Khyber Medical University, Peshawar Pakistan
}

\begin{abstract}
Objective: To determine the effects of supervised tooth brushing exercise on the oral hygiene status of school going children in Peshawar.

Study Design: Prospective observational study.

Place and Duration of Study: This study was conducted at a girl's government school in university town, Peshawar, from Jul to Dec 2018.

Methodology: All girls between age 7-10 years with mixed dentition, minimum plaque index score 2 and gingival index score 0 were included in the study. Sixty four girls were allocated into group A (intervention) and group B (control) using computer generated random numbers. Intervention group (32 subjects) was exposed to video plus supervised tooth brushing on a cast model while control group (32 subjects) only exposed to video on brushing technique. The plaque index score was recorded for oral hygiene status as outcome measure of intervention after one month.

Results: The mean age of the participants was $9.53 \pm 1.08$ years. There was statistically significant difference in the plaque scores of interventional and control group after one month $(0.3125 \pm 0.470$ vs. $1.312 \pm 0.644, p<0.001)$ as compared to baseline data $(1.90 \pm 0.296$ vs. $2.0 \pm 0.00, p=0.83)$. The difference for frequency of tooth brushing between the two groups was not statistically significant $(p=0.088)$. Parental watch during tooth brushing between the two groups was statistically insignificant $(p=0.055)$.

Conclusion: Supervised tooth brushing improved the oral hygiene status of the children therefore oral health education was effective in establishing good oral health among school children.
\end{abstract}

Keywords: Dental caries, Decayed missing filled teeth, Oral hygiene, Plaque index, Supervised tooth brushing, School children, Tooth brushing.

This is an Open Access article distributed under the terms of the Creative Commons Attribution License (https://creativecommons.org/licenses/by-nc/4.0/), which permits unrestricted use, distribution, and reproduction in any medium, provided the original work is properly cited.

\section{INTRODUCTION}

Good oral hygiene is an essential part of the overall health of an individual. Currently millions of children are affected by oral health problems because of lack of knowledge about the oral hygiene maintenance, practices and treatment services ${ }^{1}$. Evidence shows that good knowledge of oral hygiene reflects into better practices and education further improves it ${ }^{2}$. Dental caries and periodontal diseases can be initiated by the accumulation of dental plaque and were reported to be the most prevalent dental diseases of mankind ${ }^{3}$. Supervised tooth brushing programs in schools can be effective in improving the oral health of young children ${ }^{4}$. Tooth brushing with fluoride toothpaste has managed to decrease caries globally in the last decades and therefore supervised tooth brushing can be a way of assuring that the children will be sufficiently exposed to fluoride toothpaste daily ${ }^{5}$. So, an efficient preventive measure is required for school going children ${ }^{6}$.

Curnow et al concluded that supervised tooth-

Correspondence: Dr Zeeshan Kibria, Office of Research Innovation \& Commercialization, Khyber Medical University, Peshawar Pakistan

Received: 02 Jun 2020; revised received: 13 Jul 2020; accepted: 20 Jul 2020 brushing regimen along with fluoridated toothpaste had significantly reduced caries in high-caries-risk children ${ }^{7}$. They also found that the quality rather than quantity of tooth brushing matter. In this context it can be suggested that tooth brushing meticulously once a day is enough for dental caries prevention. Supervision literally means an act of direction, management or close observation. When a well-trained practitioner supervised a brushing method, they have a responsibility to guide a child to use the correct quantity of toothpaste, and make sure that each surface of teeth is brushed for adequate amount of time ${ }^{8}$. The developed countries have reduced the prevalence of poor oral hygiene and its complications through proper tooth brushing technique, flossing and mouth rinses ${ }^{9}$. There are several tooth brushing techniques, each having their merits and demerits including manual and automatic toothbrushes but the modified bass method is the most evidence-based tooth brushing technique, which is used for improving oral hygiene ${ }^{10}$. In Pakistan the habit of regular tooth brushing has been promoted mainly by business enterprises.

Some research has been done on knowledge, attitudes and practices which includes teaching the 
students on a cast models, audiovisual and instructions on child as a model but there was paucity of knowledge about the association of supervised teacher based correct tooth brushing technique and its outcome on the oral hygiene as a whole which was very important for children to understand from the very beginning. The objective of the study was to determine the effects of supervised tooth brushing exercise on the oral hygiene status of school going children in Peshawar This study would help to reduce the overall prevalence of oral diseases among the children of our community.

\section{METHODOLOGY}

This was a prospective observational study conducted in a government school of Peshawar between July 2018 and December 2018. The sample size was 64 . The sample size was calculated by software STATA 13 by taking follow up mean plaque score of $0.67(0.15)$ in the supervised teaching group and $0.52(0.16)$ in the control group, at confidence level $95 \%$ and power of the test $80^{11}$. In the study 7-10 year old female children who had plaque index not $>2$ and gingival index 0 were included in the sample and those with high decayed, missing filled teeth (DMFT) score, suffering from any systematic disease, periodontal disease or children with learning difficulties were excluded from the study. The list of children was entered in Microsoft excel and random selection of the students was done. Informed written consent was taken from the parents/guardians of the selected students and permission was obtained from the school principal. Ethical approval for the study was granted by Khyber Medical University Ethics committee (ref ltr No: Dir/KMU-EB/ES/ 000579). Prior to the allotment of students in the intervention and control group all the selected children were shown a video of the correct tooth brushing technique and then thirty two students were allotted in each intervention and control group by blocked randomization. Two teachers who were willing to participate in the study were selected and assigned to intervention group. The teachers were trained by teaching them the tooth brushing technique on a cast model and the students of intervention group were also taught the tooth brushing technique on the cast models. The plaque index score was recorded at the baseline of both groups. A closed ended questionnaire containing questions regarding the oral hygiene status was administered to both the groups to assess their oral hygiene habits.

Under supervision of the teachers assigned to the intervention group these children brushed their teeth regularly every day in front of the teachers in the morning before the commencement of their classes. After 1 month of the intervention the plaque index score was again recorded to compare the differences between the two groups.

Data was entered and analyzed by using SPSS-22 statistical software. Descriptive statistics were recorded in terms of percentages and frequencies for categorical data and means and standard deviation for numerical data. Independent t-test was used to compare the mean plaque scores between the intervention and control group. Paired t-test was used to compare the pre and post difference of plaque mean score of both the groups. The $p$-value of $\leq 0.05$ was considered significant.

\section{RESULTS}

In this study 64 children were included, 32 each in intervention and control group. Mean age of the children was $9.531 \pm 1.083$. The mean plaque score at baseline and after follow up of both the groups are given

Table-I: Comparison of mean plaque index score at baseline.

\begin{tabular}{l|c|c|c}
\hline Groups & $\mathbf{n}$ & Mean \pm SD & p-value \\
\hline Intervention group & 32 & $1.91 \pm 0.296$ & \multirow{2}{*}{0.078} \\
\hline Control group & 32 & $2.00 \pm 0.000$ & \\
\hline
\end{tabular}

Table-II: Comparison of mean plaque index score after follow up (one month).

\begin{tabular}{l|c|c|c}
\hline Groups & $\mathbf{n}$ & Mean \pm SD & p-value \\
\hline Intervention group & 32 & $0.31 \pm 0.471$ & \multirow{2}{*}{$<0.001$} \\
\hline Control group & 32 & $1.31 \pm 0.644$ & \\
\hline
\end{tabular}

Table-III: Comparison of mean difference in plaque scores between two groups.

\begin{tabular}{l|c|c|c}
\hline Groups & $\mathbf{n}$ & Mean \pm SD & $p$-value \\
\hline Supervised Group & 32 & $1.59 \pm 0.56$ & \multirow{2}{*}{$<0.001$} \\
\hline Control group & 32 & $0.69 \pm 0.64$ & \\
\hline
\end{tabular}

in table-I \& II respectively. No statistically significant differences were observed at baseline with regards to plaque index ( $p$-value 0.078). At the follow up after one month the differences observed in the plaque score were statistically significant ( $p$-value 0.000$)$. Statistically significant association $(p$-value $\leq 0.001)$ was found in the comparison of mean difference of plaque score between the two groups (table-III).

The Frequency and percentages of the oral hygiene practices in children are illustrated in table-IV.

\section{DISCUSSION}

This study showed the effects of supervised tooth brushing in improving the oral hygiene of the children. By the supervised tooth brushing children had reduction in their plaque index scores, a study conducted in 
Mexico also reveals that supervised tooth brushing at school was effective in reducing gingivitis and dental plaque $^{12}$.

Table-IV: Oral hygiene practices $(n=64)$.

\begin{tabular}{|c|c|c|}
\hline Baseline Characteristics & $\mathbf{n}$ & Percentage $(\%)$ \\
\hline \multicolumn{3}{|c|}{ Frequency of Tooth Brushing Per Day } \\
\hline Once & 13 & $(20.3 \%)$ \\
\hline Twice & 45 & $(70.3 \%)$ \\
\hline More than twice & 6 & $(9.4 \%)$ \\
\hline \multicolumn{3}{|l|}{ Cleaning Teeth With } \\
\hline Brush and toothpaste & 59 & $(92.2 \%)$ \\
\hline Dental Floss & 1 & $(1.6 \%)$ \\
\hline Toothpicks & 1 & $(1.6 \%)$ \\
\hline Other & 3 & $(4.7 \%)$ \\
\hline \multicolumn{3}{|c|}{ Tooth Brushing in Morning } \\
\hline Yes & 55 & $(85.9 \%)$ \\
\hline No & 9 & $(14.1 \%)$ \\
\hline \multicolumn{3}{|l|}{ Tooth Brushing in Noon } \\
\hline Yes & 3 & $(4.7 \%)$ \\
\hline No & 61 & $(95.3 \%)$ \\
\hline \multicolumn{3}{|c|}{ Tooth Brushing Before Going to Bed } \\
\hline Yes & 1 & $(1.6 \%)$ \\
\hline No & 63 & $(98.4 \%)$ \\
\hline \multicolumn{3}{|c|}{ Duration of Tooth Brushing } \\
\hline Less than one minute & 4 & $(6.3 \%)$ \\
\hline One minute & 19 & $(29.7 \%)$ \\
\hline Two minutes & 29 & $(45.3 \%)$ \\
\hline More than two minutes & 12 & $(18.8 \%)$ \\
\hline \multicolumn{3}{|c|}{ Parental Supervision during Tooth Brushing } \\
\hline Yes & 19 & $(29.7 \%)$ \\
\hline No & 45 & $(70.3 \%)$ \\
\hline \multicolumn{3}{|l|}{ Bleeding Gums Means } \\
\hline Don't know & 36 & $(56.3 \%)$ \\
\hline Healing gums & 6 & $(9.4 \%)$ \\
\hline Inflamed gums & 16 & $(25.0 \%)$ \\
\hline Gum recession & 6 & $(9.4 \%)$ \\
\hline \multicolumn{3}{|l|}{ Plaque Means } \\
\hline Don't know & 40 & $(62.5 \%)$ \\
\hline Inflammation of gums & 12 & $(18.8 \%)$ \\
\hline Staining of teeth & 6 & $(9.4 \%)$ \\
\hline Hard debris on teeth & 6 & $(9.4 \%)$ \\
\hline \multicolumn{3}{|l|}{ Dental Plaque Leads to } \\
\hline Don't know & 43 & $(67.2 \%)$ \\
\hline Inflammation of gums & 3 & $(4.7 \%)$ \\
\hline Staining of teeth & 5 & $(7.8 \%)$ \\
\hline Dental caries & 13 & $(20.3 \%)$ \\
\hline \multicolumn{3}{|l|}{ Visit to Dentist } \\
\hline Never & 19 & $(29.7 \%)$ \\
\hline Regularly (6-12 months) & 6 & $(9.4 \%)$ \\
\hline Occasionally & 10 & $(15.6 \%)$ \\
\hline When I need & 29 & $(45.3 \%)$ \\
\hline
\end{tabular}

Modified Bass tooth brushing technique was used in the study because this technique brought an important advancement in the oral hygiene maintenance ${ }^{11,12}$.
This statement was supported by Poyato-Ferrera et al13. They made a comparison on the efficacy in removing supragingival plaque during normal tooth brushing and a special tooth-brushing technique (modified Bass method). Their results showed that the modified Bass method is significantly superior to usual tooth-brushing technique in removing supra-gingival plaque.

This trial showed that there was no statistically significant difference in the mean baseline plaque score $\mathrm{w}$ between two groups. This showed that oral health status of both groups was similar before the intervention. The study on effectiveness of supervised tooth brushing by Damle et al and another study by Hebbal on plaque scores reported similar results to the present study ${ }^{14,15}$.

\begin{tabular}{|c|c|c|}
\hline Characteristics of Sample & R square & $p$-value \\
\hline Age & 0.04 & 0.623 \\
\hline Frequency of tooth brush & 0.002 & 0.755 \\
\hline Cleaning teeth & 0.049 & 0.078 \\
\hline Tooth brush in the morning & 0.002 & 0.729 \\
\hline Tooth brush in the noon & 0.057 & 0.057 \\
\hline Tooth brush before going to bed & 0.037 & 0.128 \\
\hline Duration of Tooth brushing & 0.006 & 0.536 \\
\hline $\begin{array}{l}\text { Parental supervision during } \\
\text { brushing }\end{array}$ & 0.011 & 0.402 \\
\hline Meaning of bleeding gums & 0.003 & 0.663 \\
\hline Gum bleeding protected & 0.076 & 0.027 \\
\hline Meaning of plaque & 0.100 & 0.011 \\
\hline Dental plaque leads & 0.183 & 0.000 \\
\hline Visit to dentist & 0.004 & 0.622 \\
\hline
\end{tabular}

The difference in mean plaque score after one month follow up was statistically significant $(p<0.001)$ between the two groups. This shows supervised tooth brushing techniques improve oral health by reducing plaque accumulation and better removal plaque. Damle et al in his had similar results to the present study ${ }^{14}$. Tsamtsouris et al in his study also showed that supervised tooth-brushing with instructions to preschool children had positive association in reducing dental plaque scores ${ }^{16}$.

A study conducted by Amin et al in Saudia Arabia on oral hygiene practices, dental knowledge, dietary habits and their relation to caries among male primary school children showed that most of the participants $(48.9 \%)$ brushed their teeth once per week ${ }^{17}$. This is in contrast to our study results because in the present study $70 \%$ children informed that they brush twice 
a day every day this could be because of more awareness of their parents regarding oral hygiene maintenance and also because certain tooth paste companies organize commercial programs in schools for the awareness of oral hygiene maintenance.

The children in the study informed that most commonly they use tooth paste and tooth brush for cleaning their teeth these results are in line with the results of another study conducted by Dixit et al on oral health knowledge and practice among indigenous Chepang school children of Nepal ${ }^{18}$. In the present study the results revealed that most of the children do not brush their teeth at noon and bed time and majority of the children brush in the morning. These results are in accordance to the study conducted by Pullishery et al on tooth brushing habits in preschool children of Mangalore, Karnataka. They reported that most of the children brushed their teeth in the morning ${ }^{19}$.

Ozbek et al reported that among school children, $41.3 \%$ brushed their teeth for $>2$ minutes, $35.7 \%$ for $<1$ minute, $21.4 \%$ for $<2$ minutes, and $1.6 \%$ for 2 minutes 20 in the present study majority of the children reported tooth brushing for 2 mins. Most of the school children $(62.5 \%)$ in the present study do not know the meaning of plaque and protection of gum and these results are similar to a study conducted by Al-Darwish about oral health knowledge, behavior and practices among school children in Qatar, they reported that $48.4 \%$ could not define the meaning of plaque ${ }^{21}$. The school children in the present study reported that they visit dentists when needed for relief from pain. Most of the time, they do not go to dentists for routine examination. A study conducted in India reported similar results that dental visits were found to be low in both urban and rural children ${ }^{22}$.

\section{LIMITATION OF STUDY}

The study was conducted on only female population. The sample size was small and further studies should be conducted on both male and female participants on relatively larger sample size.

\section{RECOMMENDATION}

There is a need of awareness programs for the maintaining of oral hygiene. Proper tooth brushing technique should be taught to the teachers also along with the students so that they can help children in maintaining their oral hygiene.

\section{CONCLUSION}

There was significant improvement in the plaque scores because of the supervised tooth brushing hence prevention from caries. Most of our school children are unaware about oral hygiene and the proper brushing technique.

\section{CONFLICT OF INTEREST}

This study has no conflict of interest to be declared by any author.

\section{REFERENCES}

1. Hamilton K, Cornish S, Kirkpatrick A, Kroon J, Schwarzer R. Parental supervision for their children's toothbrushing: Mediating effects of planning, self-efficacy, and action control. Br J Health Psych 2018; 23(2): 387-406.

2. Bhor K, Shetty V, Garcha V, Nimbulkar G. Effect of oral health education in the form of Braille and oral health talk on oral hygiene knowledge, practices, and status of 12-17 years old visually impaired school girls in Pune city: A comparative study. J Int Soc Prev Community Dent 2016; 6(5): 459.

3. Zhou N, Wong HM, McGrath C. The impact of adaptive functioning and oral hygiene practices on observed tooth-brushing performance among preschool children with special health care needs. Matern Child Health J 2019; 23(12): 1587-94.

4. Dickson-Swift V, Kenny A, Gussy M, de Silva AM, Farmer J, Bracksley-O'Grady S. Supervised toothbrushing programs in primary schools and early childhood settings: A scoping review. Community Dent Health 2017; 34(4): 208-25.

5. Dos Santos APP, de Oliveira BH, Nadanovsky P. A systematic review of the effects of supervised toothbrushing on caries incidence in children and adolescents. Int J Paediatr Dent 2018; 28(1): 3-11.

6. Sadana G, Gupta T, Aggarwal N, Rai HK, Bhargava A, Walia S. Evaluation of the impact of oral health education on oral hygiene knowledge and plaque control of school-going children in the city of Amritsar. J Int Soc Prev Community Dent 2017; 7(5): 259.

7. Curnow M, Pine C, Burnside G, Nicholson J, Chesters R, Huntington EJ. A randomised controlled trial of the efficacy of supervised toothbrushing in high-caries-risk children. Caries Res 2002; 36(4): 294-300.

8. Zacharias S, Kahabuka FK, Mbawalla HS. Effectiveness of randomized controlled field trial instructing parents to supervise children on tooth brushing skills and oral hygiene. Open Dent J 2019; 13(1): 1-2.

9. Bashirian S, Seyedzadeh-Sabounchi S, Shirahmadi S, Soltanian AR, Karimi-Shahanjarini A, Vahdatinia F. Socio-demographic determinants as predictors of oral hygiene status and gingivitis in schoolchildren aged 7-12 years old: A cross-sectional study. PloS One 2018; 13(12): e0208886.

10. Janakiram C, Taha F, Joe J. The efficacy of plaque control by various toothbrushing techniques-a systematic review and metaanalysis. J Clin Diagn Res 2018; 12(11): 1-5.

11. Srivastava N, Gupta G, Health. A comparative evaluation of efficacy of different teaching methods of tooth brushing in children contributors. J Oral Hyg Health 2013; 2013: 1-4.

12. Borges-Yáñez SA, Castrejón-Pérez RC, Camacho MEI. Effect of a school-based supervised tooth brushing program in mexico city: a cluster randomized intervention. J Clin Pediatr Dent 2017; 41(3): 204-13.

13. Poyato-Ferrera $M$, Segura-Egea J, Bullón-Fernández PJIjodh. Comparison of modified Bass technique with normal toothbrushing practices for efficacy in supragingival plaque removal. Int J Dent Hyg 2003; 1(2): 110-14.

14. Damle SG, Patil A, Jain S, Damle D, Chopal NJ, Dentistry C. Effectiveness of supervised toothbrushing and oral health edu- 
cation in improving oral hygiene status and practices of urban and rural school children: A comparative study. J Int Soc Prev Community Dent 2014; 4(3): 175.

15. Hebbal M, Ankola AV, Vadavi D, Patel KJDrj. Evaluation of knowledge and plaque scores in school children before and after health education. Dent Res J 2011; 8(4): 189.

16. Tsamtsouris A, White G, Clark EJAjodfc. The effect of instruction and supervised toothbrushing on the reduction of dental plaque in kindergarten children. ASDC J Dent Child 1979; 46(3): 204-09.

17. Amin T, Al-Abad BJIjodh. Oral hygiene practices, dental knowledge, dietary habits and their relation to caries among male primary school children in Al Hassa, Saudi Arabia. Int J Dent Hyg 2008; 6(4): 361-70.

18. Dixit LP, Shakya A, Shrestha M. Dental caries prevalence, oral health knowledge and practice among indi-genous Chepang school children of Nepal. BMC Oral Health 2013; 13(1): 20-6.

19. Pullishery F, Panchmal GS. Parental attitudes and tooth brushing habits in preschool children in Mangalore, Karnataka: A crosssectional study. Int J Clin Pediatr Dent 2013; 6(3): 156.

20. Özbek C, Eser D, Bektaş-Kayhan K, Ünür MJJoIUFoD. Comparison of the tooth brushing habits of primary school age children and their parents. J Istanb Univ Fac Dent 2015; 49(1): 33-40.

21. Harrita S, Priya VV. A questionnaire survey about oral hygiene among school children. Drug Invention Today 2019; 11(7): 1-5.

22. Batra M, Shah AF, Rajput $P$, Shah IA. Comparison of linear and zero-inflated negative binomial regression models for appraisal of risk factors associated with dental caries. J Indian Soc Pedod Prev Dent 2016; 34(1): 71-75. 